\title{
3D X-ray computerized tomography of White Etching Cracks (WEC)
}

Danielsen, H. K.; Carrasco, A. J.; Fæster, S.; Dahl, K. V.; Guzmán, F. Gutiérrez; Sauvage, P.; Jacobs, G.

Published in:

Materials Characterization

Link to article, DOI:

10.1016/j.matchar.2019.01.032

Publication date:

2019

Document Version

Peer reviewed version

Link back to DTU Orbit

Citation (APA):

Danielsen, H. K., Carrasco, A. J., Fæster, S., Dahl, K. V., Guzmán, F. G., Sauvage, P., \& Jacobs, G. (2019). 3D X-ray computerized tomography of White Etching Cracks (WEC). Materials Characterization, 150, 78-87. https://doi.org/10.1016/j.matchar.2019.01.032

\section{General rights}

Copyright and moral rights for the publications made accessible in the public portal are retained by the authors and/or other copyright owners and it is a condition of accessing publications that users recognise and abide by the legal requirements associated with these rights.

- Users may download and print one copy of any publication from the public portal for the purpose of private study or research.

- You may not further distribute the material or use it for any profit-making activity or commercial gain

- You may freely distribute the URL identifying the publication in the public portal 


\title{
3D X-ray Computerized Tomography of White Etching Cracks (WEC)
}

\author{
Danielsen HK, Carrasco AJ, Fæster S, Dahl KV, Gutiérrez Guzmán F, Sauvage P and Jacobs G
}

\begin{abstract}
This work is focused on demonstrating the possibilities of using X-ray Computerized Tomography laboratory equipment based on density measurements for making 3D maps of White Etching Crack networks in bearings. Four bearing specimens have been investigated that contained different crack morphologies which were visualized in 3D. For crack reconstruction, simple global and local threshold procedures have been successfully applied, although there are certain limitations for narrow cracks or crack tips. Specific features on the crack networks could be identified, such as crack interaction with inclusions, which was verified using microscopy characterization. By using the 3D maps as a guide for sectioning, these features were subsequently investigated with scanning electron microscopy (SEM) and chemical analysis. Through stress calculations it was demonstrated that the depth of the maximum Tresca stress and the location of WEC correlate when the real load and stress history is known. The propagation direction of the WEC is however not defined by critical plane orientation.
\end{abstract}

\section{Introduction}

White Etching Cracks (WEC) are subsurface cracks named after the deformed microstructure bordering the cracks, called White Etching Areas (WEA). The WEA consist of nano-crystalline $[1,2]$ ferrite grains formed by deformation during service, which has a white appearance in light optical microscopy after using an echtant, such as nital. The cracks are typically located within $1 \mathrm{~mm}$ from the surface of the bearing raceway and they will ultimately cause spallation leading to bearing failure, also known as White Structure Flaking (WSF). WEC is a wide spread bearing failure mode, which is in particular common for wind turbine applications, primarily in the gearbox bearings, but also in the main bearings. The WEC failure mode is known to be very aggressive and unpredictable, where premature breakdowns typically take place already after only 5-20\% of the rated lifetime [3].

In general the volume down to $1 \mathrm{~mm}$ beneath the bearing raceway experiences alternating contact stresses due to loading and unloading during every cycling rolling contact. The most commonly used theory for modelling the contact stresses in bearings is still the Hertzian theory, which considers an elastic deformation of the contacting surfaces for determining the resulting contact pressure at the interface. Roller bearings in wind turbine gearboxes are most of the operating time loaded by moderate Hertzian pressures between $1.2 \mathrm{GPa}$ and 1.6 GPa [4].

Beneath the surface a discrete volume element experiences compressive stresses $\left(\sigma_{x x}, \sigma_{y y}, \sigma_{z z}\right)$, orthogonal shear stress $\left(\tau_{0}\right)$ and unidirectional shear stress $\left(\tau_{45}\right)$. The maximum stress differs in magnitude and depth depending on the contact conditions and reach a maximum in the subsurface. With the alternating orthogonal and unidirectional shear stress reaching respectively a maximum at a depth of around $z=0.5 \cdot a$ and $z=0.78 \cdot a$ for a line contact, with $a$ being the minor semi-axis of the contact ellipse. The predicted depth at which yielding occurs during rolling contact depends on the theory used, with the Tresca shear stress criteria being frequently used. It has also been shown that the majority of microstructural alterations associated to subsurface fatigue occur at depth $z=0.75 \cdot a$ of the contact ellipse [5]. However, it has been reported that the depth of the WECs does not necessarily correlate to the depths of calculated maximum shear stresses [6].

Different morphologies for White Etching Cracks in bearings have been observed, which are possibly linked with properties of different bearing steels, different bearing configurations and different service 
exposure. In many cases WEC propagate parallel to the bearing surface (should not be considered as butterflies) and then branch out in an irregular fashion, forming crack networks which are sometimes known as irregular WEC [7] and cause failure through spallation (WSF). Tensile circumferential hoop stresses will cause WEC to propagate radially through the inner ring, causing axial cracks at the surface, also known as hairline cracks. This type of cracks are mostly seen in through-hardened bearings that can have residual tensile stresses at the surface due to the volume change during martensite transformation occurring at different times in thicker components [8]. These axial cracks are believed to originate at subsurface WEC that run parallel to the surface before propagating in the radial direction due to the high hoop stress, causing the axial cracks [9]. In this context it has been shown that a deviation of the bearing seat cylindrical form could lead to high tensile stress which eventually lead to a premature bearing failure due to WECs and axial cracks [10].

Inhomogeneities in the bearing steel such as non-metallic inclusions are stress concentration raisers that can lead to the formation of cracks and deformed microstructure around these inclusions. Such defects are frequently categorized as (two winged) butterfly cracks, extending from both sides of the inclusion in a relative straight plane. The term butterfly is traditionally used to describe both two and four winged features with cracks propagating at $30-50^{\circ}$ and $130-150^{\circ}$ (close to the angle of maximum unidirectional shear stress) from a central initiating point, usually a void or defect [11]. It has been proposed that in certain applications non-metallic inclusions could initiate the formation of WEC $[12,13]$.

WEC are normally analysed through optical microscopy or SEM, which puts $2 \mathrm{D}$ limitations on the characterization of the irregular crack networks. Using serial sectioning it is possible to recreate such crack networks in 3D using 2D techniques [12], although this method is very time consuming and destructive for the analysed specimen. It has been demonstrated that using X-ray synchrotron radiation to recreate the networks [13] is possible, however such techniques require specialized, highcost large scale facilities where measurement time may not be readily available. Recent advances with 3D X-ray Computerised Tomography (XCT) allow for 3D high resolution imaging of metallic specimens with laboratory equipment, see figure 1 . Reconstruction of XCT data provides 3D density maps that allow detection of small density differences in the bulk steel material, such as the presence of crack networks or non-metallic inclusions. The main limitation is the size of the specimen, which dictates the level of resolution that can be achieved. This is especially critical for investigating narrow subsurface cracks, while cracks opened to the surface are usually wider and more easily characterized.

The purpose of this paper is to demonstrate that current X-ray Computerized Tomography (XCT) equipment is suited for studying the morphology of WEC networks. It was also attempted to correlated the depth of the observed WEC with the calculated maximum Tresca stress and the critical plane orientation. Three different wind turbine gearbox bearings with different WEC morphology were investigated in this work, as well as WEC that were formed in a laboratory test rig under accelerated conditions. 


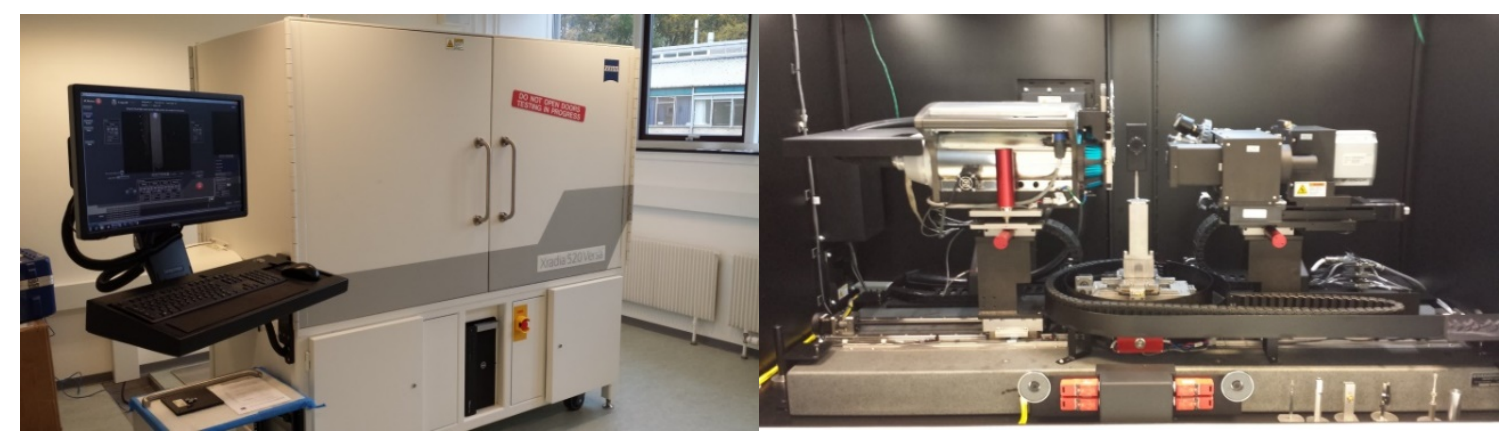

Figure 1: (a) Zeiss Xradia 520 Versa used for 3D X-ray Tomography. (b) Small rod specimen rotating $360^{\circ}$ between $X$-ray source and detector.

\section{Experimental method}

\section{$\underline{X \text {-ray computerized tomography }}$}

The laboratory equipment used was a Zeiss Xradia 520 Versa capable of achieving a spatial resolution down to $0.7 \mu \mathrm{m}$ and a theoretical minimum achievable voxel size down to $70 \mathrm{~nm}$ under ideal circumstances. The X-ray energy used was polychromatic up to $160 \mathrm{kV}$ from a tungsten target, and projections were acquired during a full sample rotation of $360^{\circ}$ on a $2 \mathrm{k} \times 2 \mathrm{kCCD}$ with a pixel size of $13.5 \mu \mathrm{m}$. 3D maps were reconstructed by a Feldkamp algorithm for cone beam reconstruction [14] to $2 \mathrm{k} \times 2 \mathrm{k} \times 2 \mathrm{k}$ pixel volumes. The standard Zeiss Xradia procedure where followed for selecting the appropriate energy and source filter to tune the $\mathrm{X}$-ray energy spectrum for the individual samples. The exposure time for each projection was chosen as high as possible without saturating the detector and were between 10-20 sec. for the different samples. Several standard filtering algorithms were tested, however it was decided not to use any filtering when analysing the data in order not to smear out the very narrow crack lines. The crack networks were segmented using a global intensity threshold value and visualized using Avizo 3D software for materials science.

In modern micro tomography systems the spot size of the X-ray source has been minimized. This makes it possible to place the sample very close to the source to achieve very high geometric magnifications and at the same time minimizing the geometric blurring. Some XCT systems utilize both geometrical and an optical magnification in order to minimize the spot size dependency on the achievable resolution. The small distance between the object and the $\mathrm{X}$-ray source results in the high magnification but also constitute the main limitation as the sample has to be relatively small in order to image the whole sample on the detector.

Global thresholding is the most simple segmentation technique, and is ideal for images with a bimodal intensity distribution [15]. If the sample contains more than two phases then a region based segmentation method such as the watershed method would normally give better results. However, the watershed method has disadvantages like sensitivity to noise and being poor at detecting narrow structures which are of critical importance when investigating crack networks. The disadvantage of the global threshold method is the risk of removing or breaking up cracks into smaller parts. Detailed schemes, involving multiple image processing steps, need to be developed for the dataset on hand to overcome the problem with breaking cracks due to segmentation. An example of such an image processing method can be found in [16].

In this work simple methods were tested for easy reconstruction of crack networks and inclusions. Global thresholding was chosen for larger cracks using a single threshold value, which gives a very fast 
visualization of the crack network and inclusions. This method only allows visualization of clear features such as wide cracks and inclusions, while narrow cracks cannot be visualized without introducing significant noise. For visualizing narrow cracks, a semi-automatic local thresholding was done utilizing a magic wand tool on several sub-volumes of a feature manually receiving an individual threshold value. In cases where features overlap it can be hard to tell them apart, such as when cracks passing through inclusions or voids these features would be segmented as part of the crack. In order to visualize the inclusion interaction with cracks, the inclusions were selected by eye using an individual local threshold value and given a separate color. In order to distinguish inclusions from the cracks, which had lower threshold values and thus engulfed them visually, the inclusions were enlarged by cube dilation in 3D considering the neighbouring 26 voxels with a half-kernel size of 2 . Thus, the size of the inclusions are overestimated but their location is correct and the interaction with the crack network can be visualized in 3D.

XCT was applied at positions where WEC had already been located, using visual inspection for axial cracks, and optical microscopy for irregular WEC and butterfly-like cracks. Samples were extracted based on the position of the axial cracks in such a way that they would contain a complete WEC network, but for the other irregular WEC and butterfly-like cracks only part of the WEC networks were contained in the specimens due to cuts already being made during optical microscopy. The XCT specimens were cut out with a rod-shaped geometry, having a cross section between $\approx 0.5 \times 0.5 \mathrm{~mm}$ to $\approx 5 \times 5 \mathrm{~mm}$, the length was arbitrary. For the larger WEC reconstructed volumes, such as in the case of axial cracks, several scans from the same specimen were stitched together to form one large 3D map encompassing the entire crack. The stitching was performed using the Zeiss Xradia standard procedure for vertical stitching where the projection data is stitched before the reconstruction is performed.

\section{Specimen information}

All investigated bearings were mode from through hardened $100 \mathrm{Cr} 6$ bearing steel variants. Three different bearings from wind turbine gearboxes, which had been affected by WEC formation were used as demonstration of the XCT technique, as well as a bearing from a laboratory test rig used for accelerated WEC tests for comparison, see table 1.

Table 1: Bearings investigated in this work

\begin{tabular}{|c|c|c|}
\hline Specimen & WEC-Morphology & Bearing type \\
\hline A & Irregular WEC & Axial bearing, FE8 type test rig \\
\hline B & Axial WEC & Intermediate stage tapered bearing, wind turbine \\
\hline C & Butterfly-like WEC & High speed shaft ball bearing, wind turbine \\
\hline D & Irregular WEC & Planetary stage radial bearing, wind turbine \\
\hline
\end{tabular}

Specimen A is from a cylindrical roller thrust bearing run in a laboratory FE8 test rig. This test rig has been used in previously published work [17] for demonstrating the reproducibility of WEC induced failures by simultaneously testing two cylindrical roller thrust bearings of type 81212, which are statically loaded by a plate spring package. In order to avoid a chemical influence a polyamide cage (PA 66) was used instead of the usual brass cage. The test was conducted with a constant oil flow of $0.25 \mathrm{l} / \mathrm{min}$ using a fully formulated, commercially available mineral oil with a viscosity grade of ISO VG 100. The test was conducted under constant conditions at $80 \mathrm{kN}(1.9 \mathrm{GPa}$ using fifteen $11 \times 11 \mathrm{~mm}$ rollers), $300 \mathrm{rpm}$ and $100^{\circ} \mathrm{C}$ until flaking occurred. As already shown in [17], these conditions lead to 
reproducible WEC induced failures with a Weibull $\beta$-value of 2.4. Ultrasonic measurements and optical microscopy showed extensive presence of subsurface WEC, further information can be found in [18].

Specimen B was from the inner ring of a through hardened tapered roller bearing belonging to a 2 MW class turbine. The tapered roller bearings were installed pairwise in the helical intermediary stage of a three-stage planetary-helical design gearbox, with an inner bore around $190 \mathrm{~mm}$. Detailed specifications are not given for reasons of confidentiality. The studied inner ring was from the bearing placed closest to the rotor side. The bearings were removed prematurely after only few years of service and axial cracks could be observed on the raceway surface during visual inspection on both inner rings (rotor side and generator side).

Specimen $\mathrm{C}$ was a failed four-point-ball bearing with a two-piece inner ring from a $1 \mathrm{MW}$ class gearbox, with an inner bore around $140 \mathrm{~mm}$. The bearing was designed to take up axial loads on the high speed shaft just before the generator. The bearing was removed after five years due to an increase in structural vibration that was registered by the condition monitoring system even though the calculated nominal life according to DIN 281 was more than 200,000 h. After removal flaking was observed on the bearing inner rings.

Specimen D was from the inner ring of a failed radial Cylindrical Roller Bearing (CRB) belonging to a 3 MW class turbine. The radial CRB was installed in the planetary stage of a three-stage planetary-helical design gearbox, with an inner bore around $200 \mathrm{~mm}$. The bearings were removed prematurely after six years of service and spallation could be observed on the raceway surface during visual inspection of the inner rings.

\section{Calculation of subsurface stresses}

For the investigated bearings, the contact geometries, the normal contact stresses and the friction coefficient are used as input for the computation of discrete contact stress and subsurface stress distributions. In contrast to specimen A the load history of specimens B, C and D is unknown. Therefore, it is assumed that the recommended maximum contact pressure for wind turbine bearings according to the wind turbine design standard IEC 61400-4 [19] has been adequately complied with. This limits are found in table 2 and are assumed for the calculation of sub-surface stresses.

Table 2: Recommended maximum contact pressure for wind turbine bearings according to [19]

\begin{tabular}{|c|l|c|}
\hline Specimen & Gearbox stage & Recommended maximum contact pressure \\
\hline B & Intermediate stage & $1,65 \mathrm{GPa}$ \\
\hline C & High speed shaft & $1,30 \mathrm{GPa}$ \\
\hline D & Planetary stage & $1,50 \mathrm{GPa}$ \\
\hline
\end{tabular}

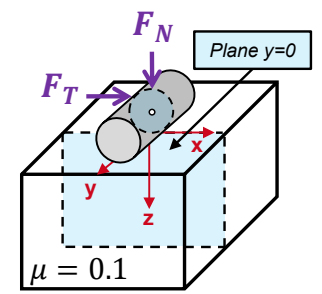

Figure 2: Coordinate system and representation plane of the computed Tresca stress distribution. 
Within the framework of this work, contact surfaces are assumed to be smooth and remain elastic. Furthemore, the rolling element profiles are based on DIN 16281 [20] and the relocation of the subsurface stresses as a result of a traction force at the surface is consider through a constant friction coefficient of 0.1, which according to [21] is typical for running rolling bearings, see Figure 2 .

The computation is achieved with a semi-analytical method described in [22]. The contacting bodies are assimilated to half-spaces, and their contact surfaces meshed into a $200 \times 200$ elements grid. In case of the specimen $A$ this results in elements with size of $4.1 \mu \mathrm{m}$ in rolling direction and $110 \mu \mathrm{m}$ in contact width direction. The resulting relative normal contact stress and shear contact stress distribution in rolling direction for specimen A are represented in figure 3 a) and b) respectively. For reasons of confidentiality, the geometries and the contact stress distributions are not given for specimens $\mathrm{B}, \mathrm{C}$ and $\mathrm{D}$.
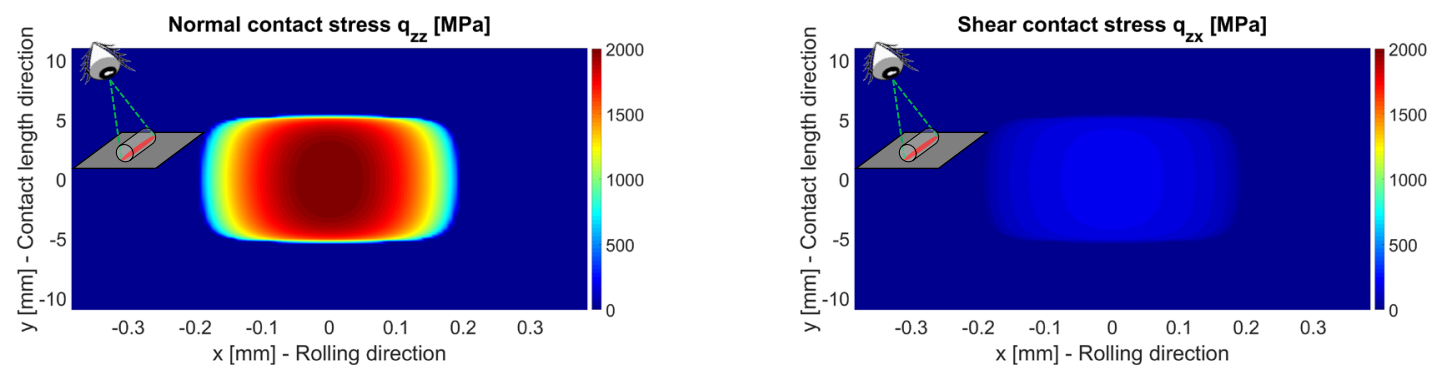

Figure 3: (a) Elastic normal contact stress distribution for specimen $A$. (b) Elastic shear contact stress distribution in rolling direction for specimen $A$

From the two-dimensional discrete normal and shear contact stress distributions, the corresponding three-dimensional discrete subsurface stress tensor distributions are computed using the method described in [23]. The contacting bodies was meshed into a $200 \times 200 \times 200$ elements grid. In case of the specimen $A$ this results in elements with a size of $4.1 \mu \mathrm{m}$ in rolling direction, $110 \mu \mathrm{m}$ in contact width direction and $10 \mu \mathrm{m}$ in depth direction. The Tresca stress, calculated from the stress tensors, is represented in figure 4 for each bearing in the plane $y=0$, which is the symmetry plane of the roller in contact width direction and the plane in which the Tresca stress reaches its maximum. For reasons of confidentiality, the $x$-axis (rolling direction) is normalized by $b$, which is half the length of the contact surface dimension in $\mathrm{x}$-direction. The depth of maximum Tresca stress in the plane $\mathrm{y}=0$ was calculated to be $146 \mu \mathrm{m}$ for specimen $\mathrm{A}, 340 \mu \mathrm{m}$ for specimen $\mathrm{B}, 183 \mu \mathrm{m}$ for specimen $\mathrm{C}$ and $430 \mu \mathrm{m}$ for specimen D. Due to the presence of the traction force and the resulting shear stress on the contact surface, the largest Tresca stress increases and shifts towards the surface. In case of specimen A by considering a traction force the Tresca stress depth is reduced in approximately $5 \%$.

It is well known that rolling contact generates a non-proportional stress state in the contacting bodies. Under this state of stress, a crack tends to initiate in the plane of largest shear strain amplitude, i.e. the critical plane [24]. From the discrete sub-surface stress tensor distribution, the position of the largest shear strain amplitude as well as the orientation of the critical plane is computed based on [25] for all the specimens. The position of the largest shear strain amplitude does not coincide with the position of the maximum Tresca stress in any specimen. Moreover, for all the specimens, the critical plane orientation in the inner ring is described by an angle $\theta$ in the range $\left[84^{\circ} ; 88^{\circ}\right]$ towards the rolling direction. This is due to the similar sub-surface stress states, despite their different magnitudes, for the used profiles, friction coefficient and material. 

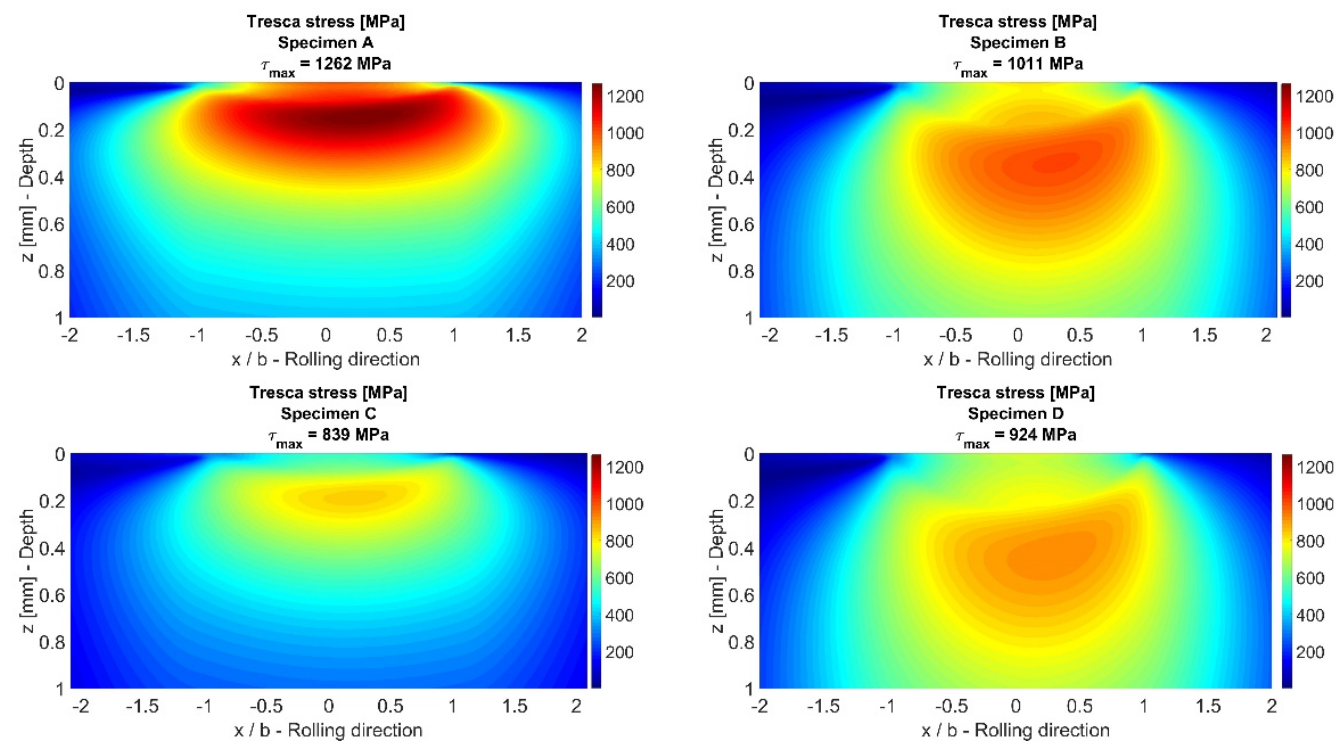

Figure 4: Subsurface distribution in the inner raceway of the Tresca stress in the plane $y=0 \mu \mathrm{m}$ for (a) specimen $A$ with depth of maximum stress at $146 \mu \mathrm{m}(b)$ specimen $B$ with depth of maximum stress at $340 \mu \mathrm{m}$ (c) specimen $C$ with depth of maximum stress $184 \mu \mathrm{m}$ (d) specimen $D$ with depth of maximum stress at $430 \mu \mathrm{m}$.

\section{Results}

\section{Irregular WEC network}

Investigations of Specimen A revealed extensive formation of branching WEC networks below the surface characterized in [18], sometimes referred to as irregular WEC. Different crack network sizes were observed with many subsurface WEC located 100-200 $\mu \mathrm{m}$ below the surface, corresponding well with the calculated maximum Tresca stress at $146 \mu \mathrm{m}$, and in some areas spallation had taken place. For this work a WEC was chosen, which had connected to the surface and was in the stage just before spallation. The surface connection allows the main crack to open up, making it clearly visible using XCT due to the larger width of the crack. Figure 4 shows a comparison between optical microscopy and $X C T$, with the XCT image being just below the cut surface on which the optical image was acquired. The Vickers hardness indent in the upper left corner on the XCT scan was used to identify the location of interest during specimen preparation. The images in figure 5 show that XCT can obtain close to the same resolution as standard optical microscopy, with the advantage being the 3D aspect of the measurements. Since the main crack is very wide it is easily visible, and while the side cracks are much narrower they can still be observed using XCT. 


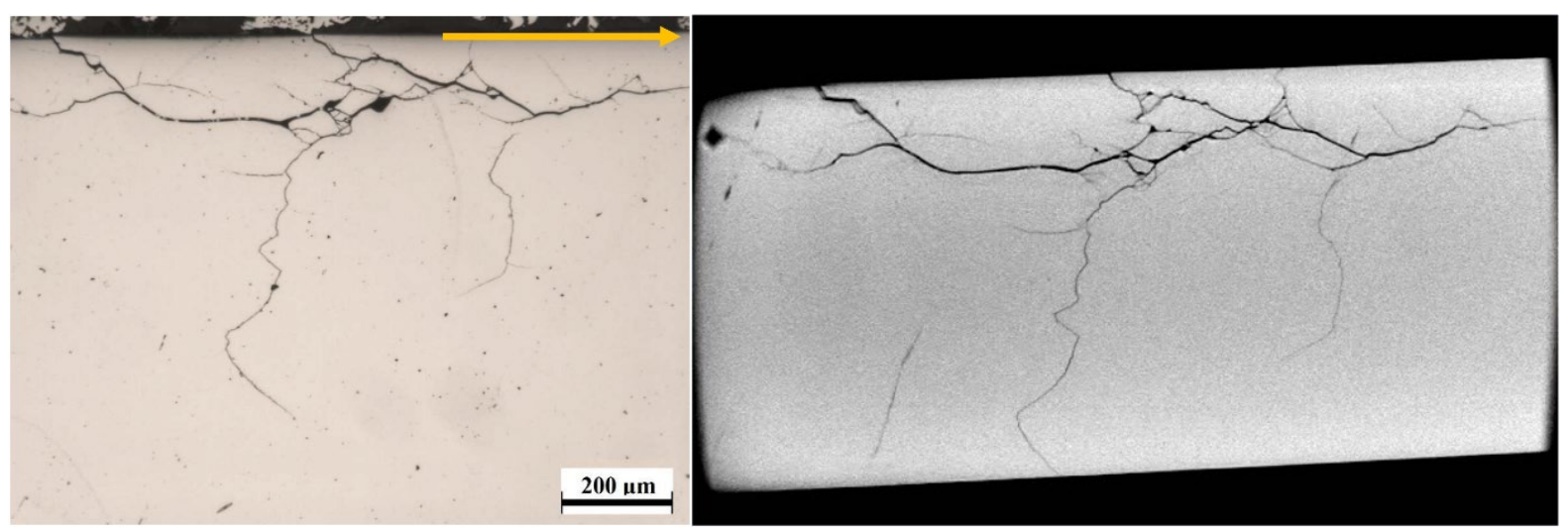

Figure 5: WEC from specimen A, FE8 test rig thrust bearing, approximately same areas are shown in both images. (a) Optical image of sample surface. The arrow indicates the rolling direction. (b) Virtual slice through tomographic reconstruction just below the sample surface of figure 5a. A Vickers hardness indent used for orientation is seen in the upper left corner of the sample, voxel size is 0.70 $\mu \mathrm{m}$. Viewed along rolling direction, calculated maximum Tresca stress $146 \mu \mathrm{m}$ and critical plane at $85.0^{\circ} \pm 0.7$.

Figure 6 shows a 3D reconstruction of the scanned volume using a single manually set global threshold value, other than that the reconstruction procedure was automatic. The steel matrix is set to be transparent while anything with lower density is in yellow, this includes both cracks and inclusions. MnS type elongated inclusions up to hundreds of microns long were observed in the axial bearing, being easily identified due to the elongated shape of the inclusions. The representation of the 3D reconstruction in figure 6 illustrates how the MnS inclusions are preferentially aligned in a specific direction, most likely due to the manufacturing process. Both size and shape distribution of such inclusions are easily obtained using XCT due to their relative large thickness (compared to cracks). The estimation of the true size of such elongated inclusions is difficult using 2D microscopy unless the specimen is cut exactly along the longitudinal direction of the inclusion. There does not seem to be any systematic correlation between the WEC propagation and the elongated MnS inclusion, although it can be imagined that MnS could have acted as crack initiators. The WEC itself is much more narrow than the inclusions, and although the automatic segmentation procedure is based on a constant global threshold value, it is sufficient for 3D imaging of the open crack, with the narrower side branches posing more of a challenge to map. In figure 6 there are two WEC side branches, which penetrate deeper into the bearing, where the automatic segmentation still is possible. However, close to the crack tips the contours start to become discontinuous as the crack narrows, being close to the limitation of the global threshold segmentation technique. For better imaging of such narrow cracks, a local threshold segmentation procedure could be used where an operator manually helps the software to identify the crack by setting separate threshold values for different portions of the crack. 


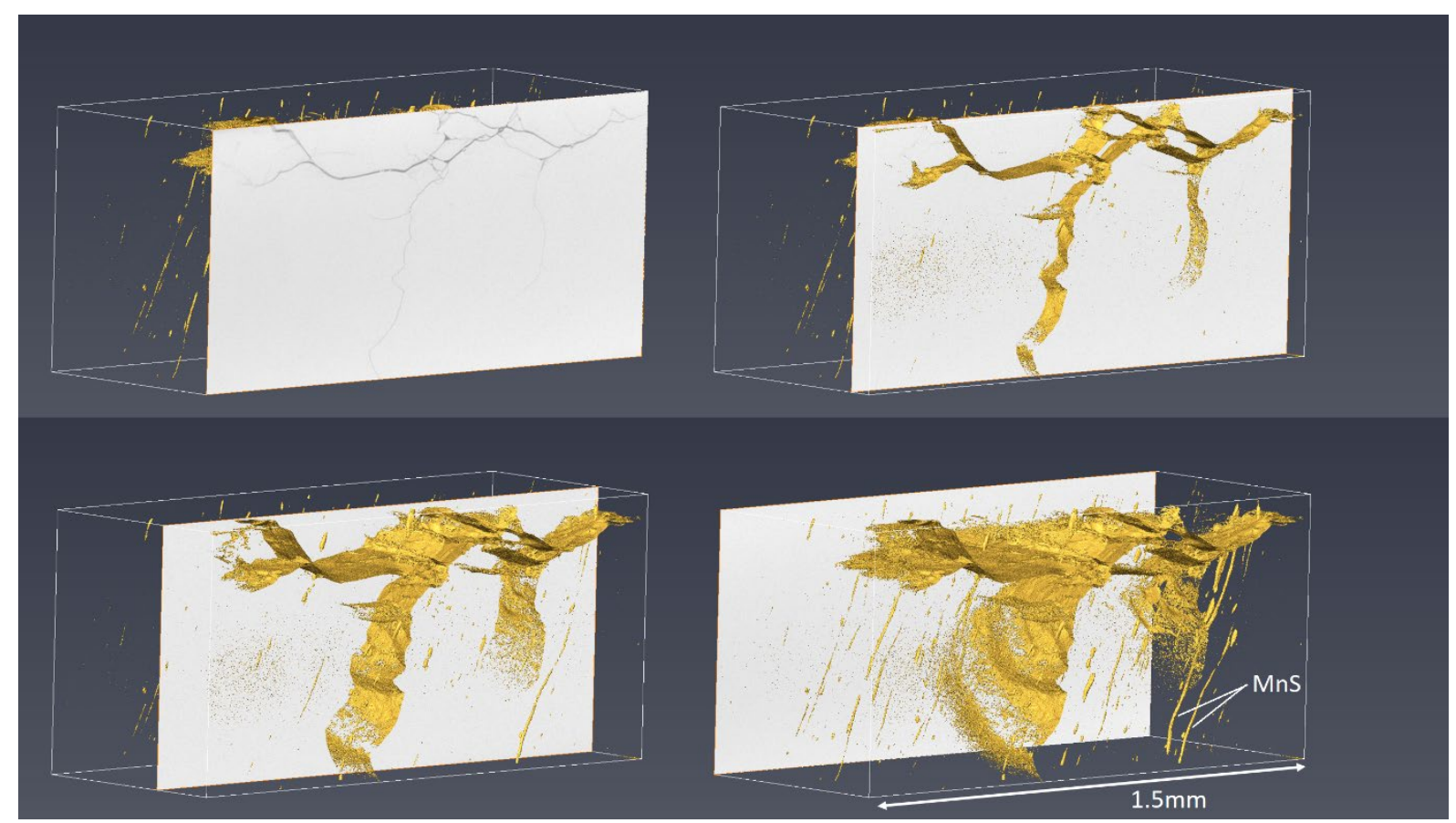

Figure 6: Illustration of four different virtual sections through the tomographic reconstructed volume, from same area as shown in figure 5. The 3D crack network and inclusions are highlighted in yellow, for clarity the raceway surface of the bearing is not shown, voxel size is $0.70 \mu \mathrm{m}$.

\section{$\underline{\text { Axial cracks }}$}

Specimen B showed extensive axial cracks on the raceway surface of the inner ring, see figure 7 . The inner ring contained around 50 cracks visible by eye, typically $10-40 \mathrm{~mm}$ long and very straight along the axial direction. A section containing one of the WEC was cut out and scanned using XCT. Since the hairline crack was relatively big and opened up at the surface, a relatively large sample size could be scanned. The crack was over $30 \mathrm{~mm}$ long and extended down through the entire scanned volume. Due to the length of the crack, several scans were made on the specimen and the scans were then stitched together for a complete 3D overview of the crack. The total reconstructed volume was of the size 33 $\mathrm{mm} \times 4.5 \mathrm{~mm} \times 1.5 \mathrm{~mm}$, see figure $7 \mathrm{a}$.

The XCT scan revealed a completely straight crack close to the surface of the bearing raceway, going straight down vertically. However, further beneath the surface, the crack contained ledges that ran roughly parallel to the raceway surface before continuing further down into the bearing, also in a straight down vertical fashion, see red line in figure $8 \mathrm{~b}$. Two major separate ledges could be identified within the scanned crack, see yellow lines in figure $8 \mathrm{~b}$, being roughly located in the middle of the crack and connected by the vertical portion of the crack. The axial crack most likely started as two separate subsurface WECs at these ledges, as WEA generally observed at the ledges (figure 7b) indicates these axial cracks are not caused by rapid cleavage but rather by fatigue crack growth. After reaching a critical length, they then propagate in axial direction up towards the raceway and down towards the shaft due to tensile circumferential hoop stresses. There is some limited branching of the crack, in particularly at the edge of the two ledges where the crack changes between vertical and horizontal directions. The ledges were about $6 \mathrm{~mm}$ long in the axial direction and $1 \mathrm{~mm}$ at maximum width, located 1-2 $\mathrm{mm}$ below the raceway surface. This is however much deeper than the calculated maximum Tresca stress at a depth of $340 \mu \mathrm{m}$, although some ledges with WEC were observed around this depth, see figure $7 \mathrm{~b}$. 


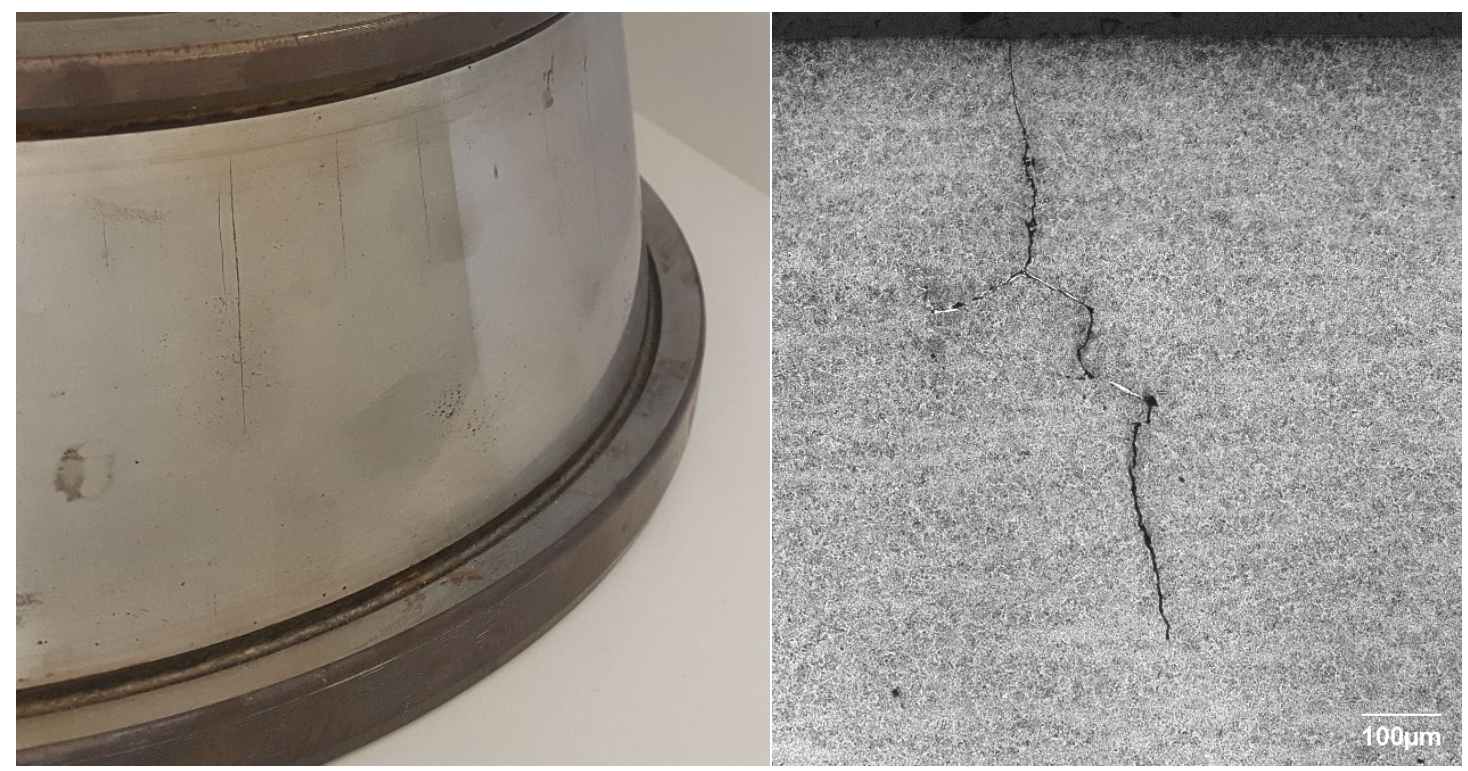

Figure 7: Specimen B. (a) Around fifty 10-40 mm long straight axial cracks were observed on the raceway. (b) Small axial crack propagating almost vertically down, several ledges that contain WEA are present. Viewed along rolling direction, calculated maximum Tresca stress $340 \mu \mathrm{m}$ and critical plane at $86.75^{\circ} \pm 0.75$.

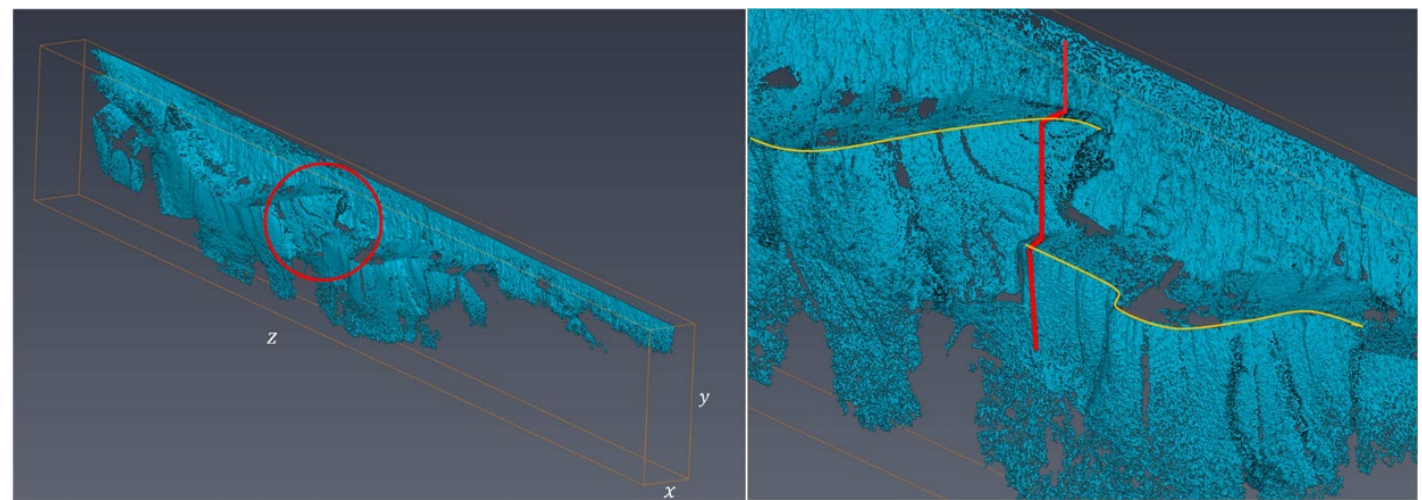

Figure 8: XCT of a large axial crack from specimen $B$, voxel size is $3.96 \mu \mathrm{m}$. (a) $33 \mathrm{~mm} \times 4.5 \mathrm{~mm} \times 1.5$ $\mathrm{mm}$ stitched tomographic reconstruction of an axial crack highlighted in blue. $Z$ is the axial direction, $Y$ is the radial direction and $X$ is the rolling direction. (b) Magnification of the location in the red circle in figure 8a, showing two ledges beneath the raceway surface.

\section{Butterfly-like cracks}

Specimen C contained a multitude of what could be described as large butterfly-like type WECs, essentially straight cracks lying at a specific angle to the bearing raceway. Cross sectional analysis using light optical microscopy revealed regular WECs inclined approximately $20^{\circ}$ to the raceway surface in an axial cross section, see figure 9 , which is a shallower angle than the $30-50^{\circ}$ normally associated with butterflies. The regular appearance of the WEC (no branching) as well as the frequent appearance of inclusions inside the WEC point toward a failure governed by the stress field and possibly overload. Most cracks were centered in depths of around 200-300 $\mu \mathrm{m}$, which is slightly more than the calculated depth of $184 \mu \mathrm{m}$ for the maximum Tresca stress, which could be could be interpreted as a further indication of overload and the resulting displacement of the maximum Tresca stress deeper in the material. Some of the cracks were in contact with the surface with some spallation occurring, however, most of the cracks were of subsurface nature, making it difficult to characterize using XCT scans due to the narrowness of the cracks. 


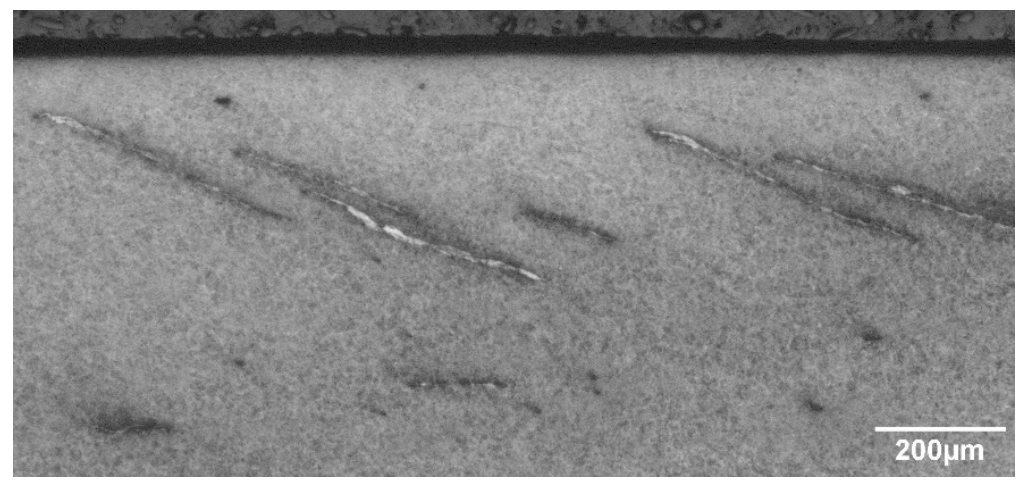

Figure 9: Reflected light microscopy image of specimen $C$ where the WEC are inclined to the raceway surface in axial cross section. Viewed along rolling direction, calculated maximum Tresca stress 184 $\mu m$ and critical plane at $86.7 \pm 0.8^{\circ}$.

Figure 10 and 11 show optical imaging and a 3D tomographic reconstruction of several subsurface cracks located at roughly the same depth and having roughly the same angle to the surface. While the cracks were imaged using manually set local thresholds in the segmentation procedure, with an operator assisting the software in identifying the crack, there were still some portions of the cracks that were too narrow for 3D imaging. Thus, the cracks appear to be discontinuous having gaps in them or appear as several smaller cracks lying on the same plane with small gaps between them. This is not a necessarily a true feature, but could be caused by limitations of the XCT technique so it must be assumed that all cracks lying on the same plane are connected. Using a time consuming manual paint process of the crack in each slice could have improved the result as the human eye is better at identifying cracks than the software is, however, this was not within the scope of this work. Several inclusions being in direct contact with the cracks were detected, these have been highlighted in white in figure 10. There were many more inclusions, which were not in contact with any crack, these are not highlighted for clarity. The main butterfly-like cracks shown in figure 11 all contain inclusions. Some of the cracks at the edge of the scan do not, but these cracks most likely continue outside of the scanned volume. In general the inclusions in this sample did not have an elongated shape as the ones observed in the axial bearing (figure 5).
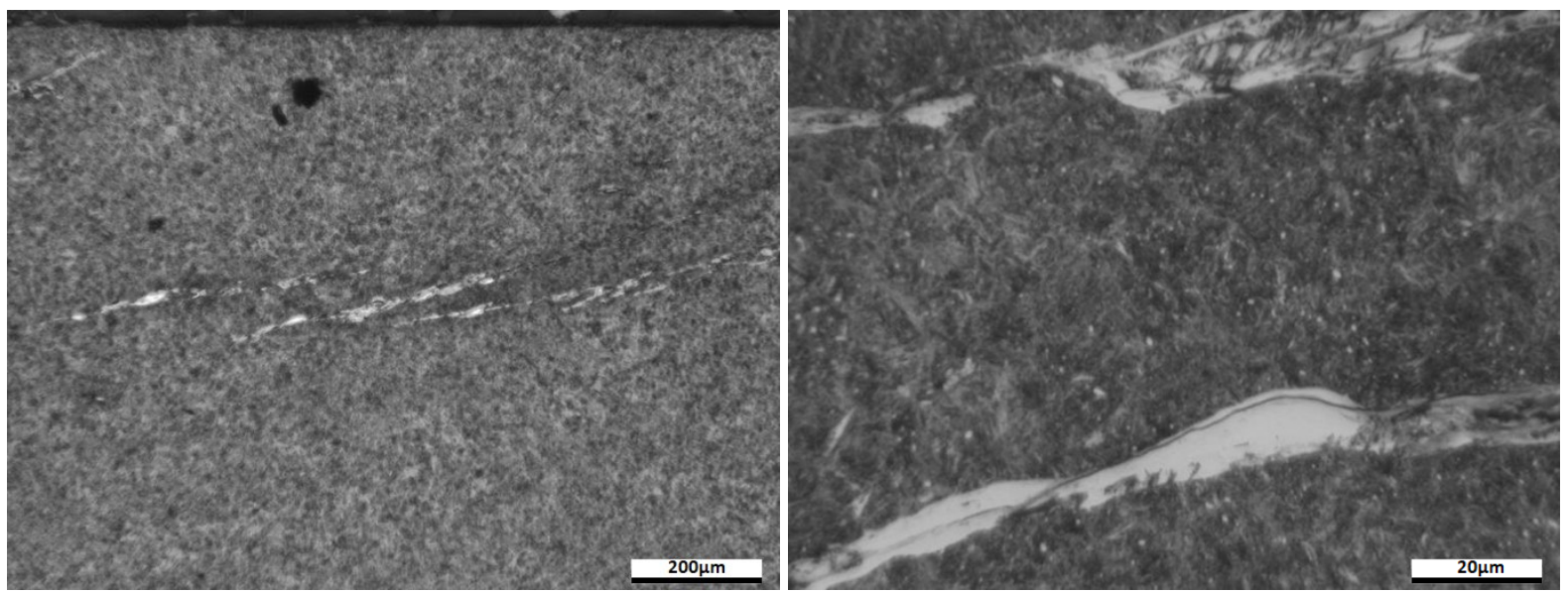

Figure 10: Optical imaging of butterfly-like cracks from a specimen C. (a) Several butterfly-like cracks located below the raceway chosen for XCT investigation. (b) Magnification of figure 10a, nital etching makes the WEA clearly visible. 

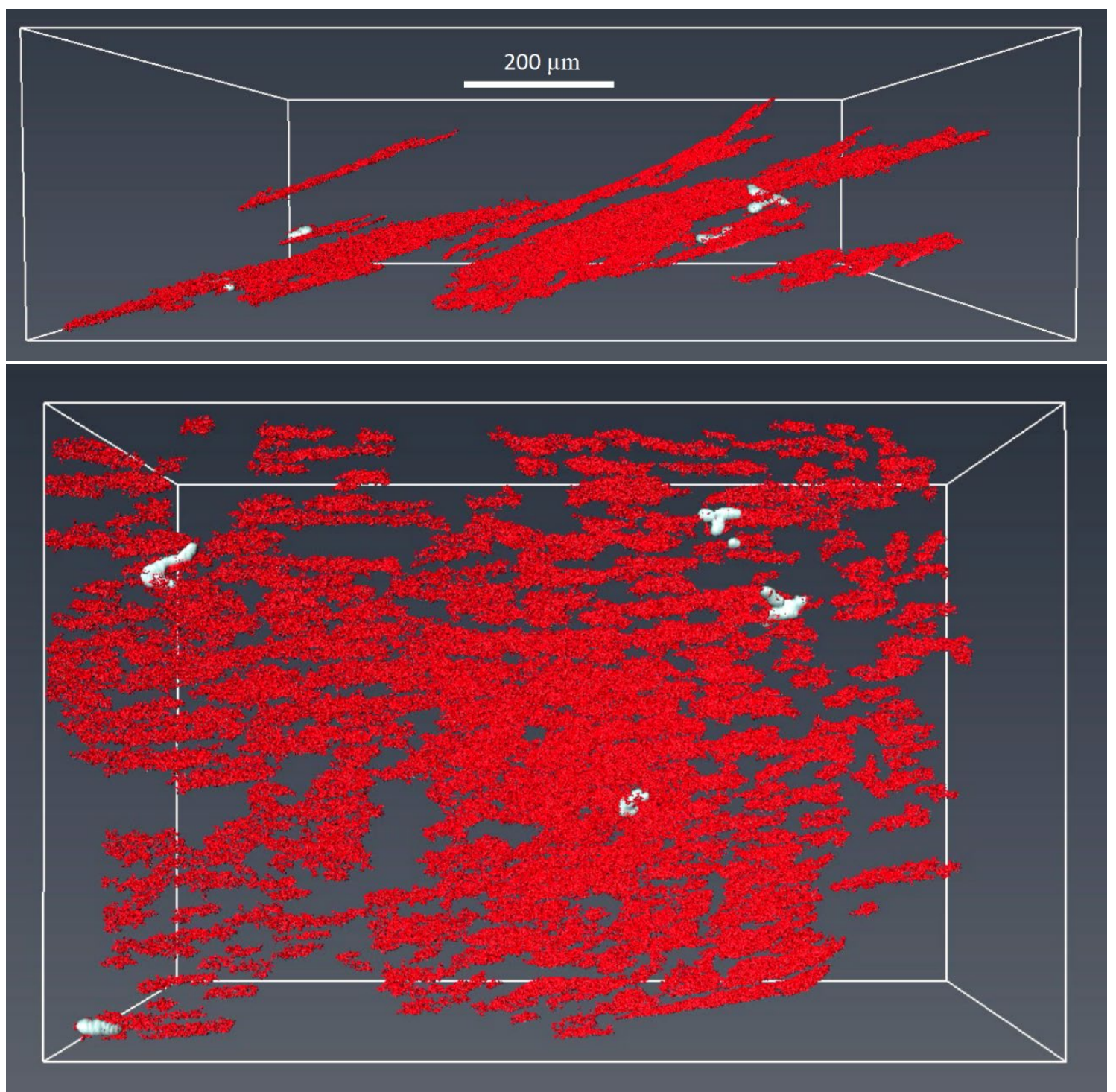

Figure 11: Tomographic reconstruction of butterfly-like cracks and inclusions from the location in figure 10a. The cracks are in red and inclusions are in white, voxel size is $0.60 \mu \mathrm{m}$. Inclusion size has been slightly enlarged for better visualization. (a) Side view of the same area seen in figure 10a. (b) Top view.

Sectioning of this sample and subsequent optical microscopy was performed to verify the results of the XCT scan, especially to verify if the observed inclusions truly were inclusions. Using the 3D maps for orientation, the locations imaged with XCT could be reached without using serial sectioning, but by grinding down to a pre-determined depth. It was confirmed that the features observed in XCT could also be seen using optical microscopy, see figure 12, including some of the highlighted inclusions in figure 11. It was also confirmed using SEM that cracks ran through or along these inclusions, see figure 13a, EDS analysis confirmed that they were of the MnS type. The XCT had a resolution close to standard optical microscopy, however SEM gave a far superior imaging of the smaller cracks and crack tips. SEM imaging indicated that cracks with a width down to $0.5 \mu \mathrm{m}$ were clearly visible in XCT. SEM also showed the presence of discontinuities in the butterfly-like cracks, figure $13 \mathrm{~b}$, and thereby indicating there might actually be some gaps in the cracks in the butterfly-like cracks. 


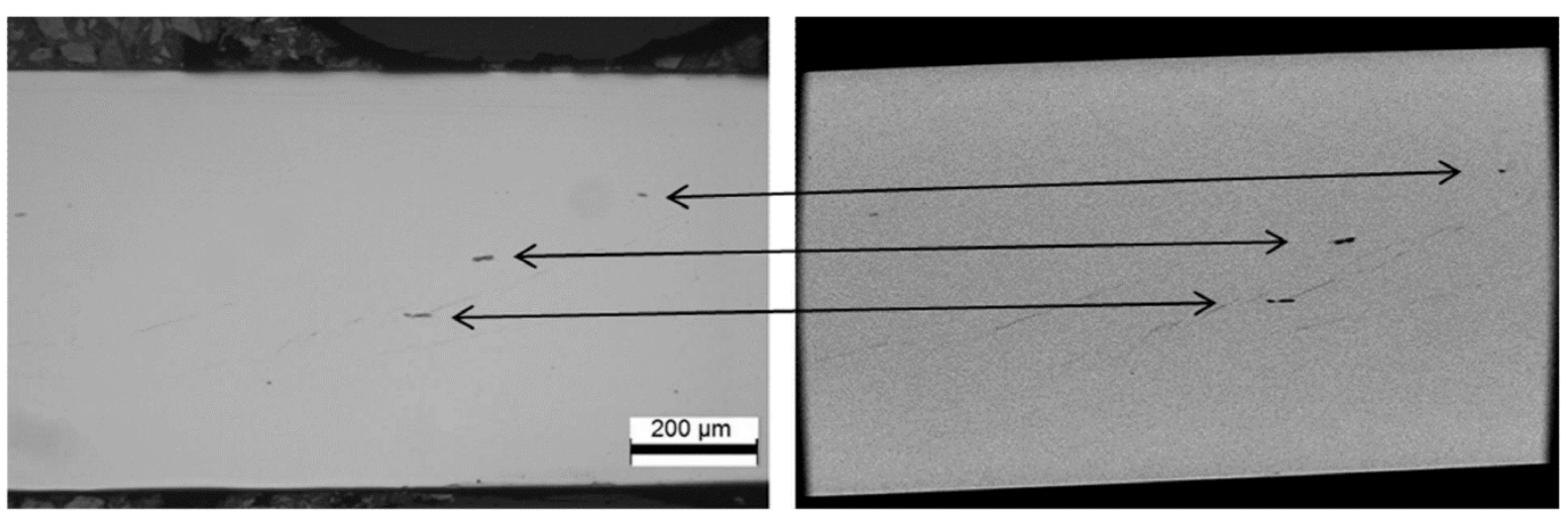

Figure 12: Sectioning down to predetermined depth from figure 11 shows the crack interacting with inclusion. (a) Optical microscopy of the butterfly-like cracks and (b) corresponding XCT slice, voxel size is $0.60 \mu \mathrm{m}$.

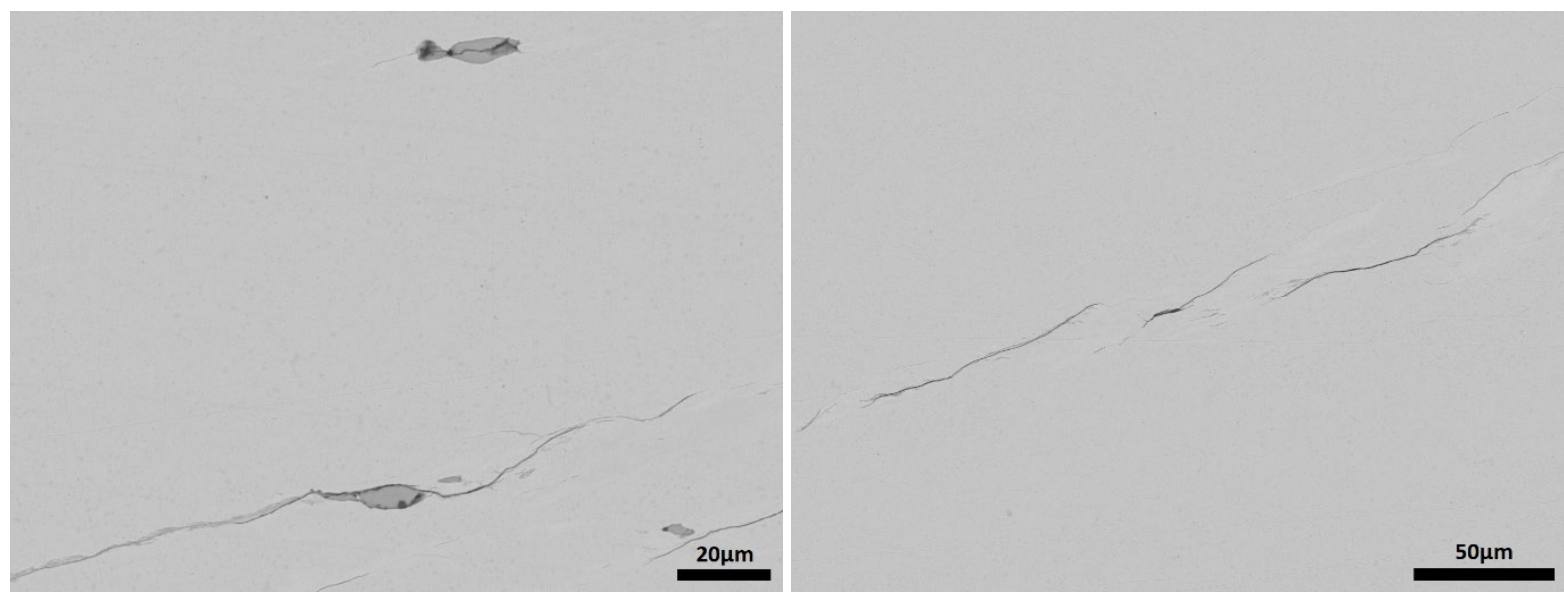

Figure 13: SEM taken from same volume and direction as in figure 11a. (a) Inclusion interaction with cracks. (b) Discontinuous section of a butterfly-like crack.

\section{Discussion}

Some crack morphologies are somewhat more complicated than other WEC morphologies, as illustrated by the examples of irregular WEC networks and axial cracks given in this paper, thus 3D scanning using XCT is a very useful tool for getting a more complete overview of these crack networks. Specimen $A$ and $D$ were used for comparison between irregular WEC formed during laboratory tests and irregular WEC formed in wind turbines. Some differences in crack network morphology would be expected when comparing irregular WEC from a small FE8 test rig axial bearing run under controlled laboratory conditions with constant load, speed and with automotive lubricant to an irregular WEC from a full sized wind turbine gearbox planetary stage radial bearing run under wind turbine service conditions with wind turbine lubricant. The calculated maximum Tresca stresses are also quite different, being $146 \mu \mathrm{m}$ for specimen A and $430 \mu \mathrm{m}$ for specimen D. However, figure 14 shows that the two cases of WEC look remarkably similar, even though the conditions for their formation have been very different. This suggests the root cause or damage mechanisms are similar for these two cases, which may or may not be the case, but at least the overall appearance shows striking similarities in crack propagation and damage progression. 

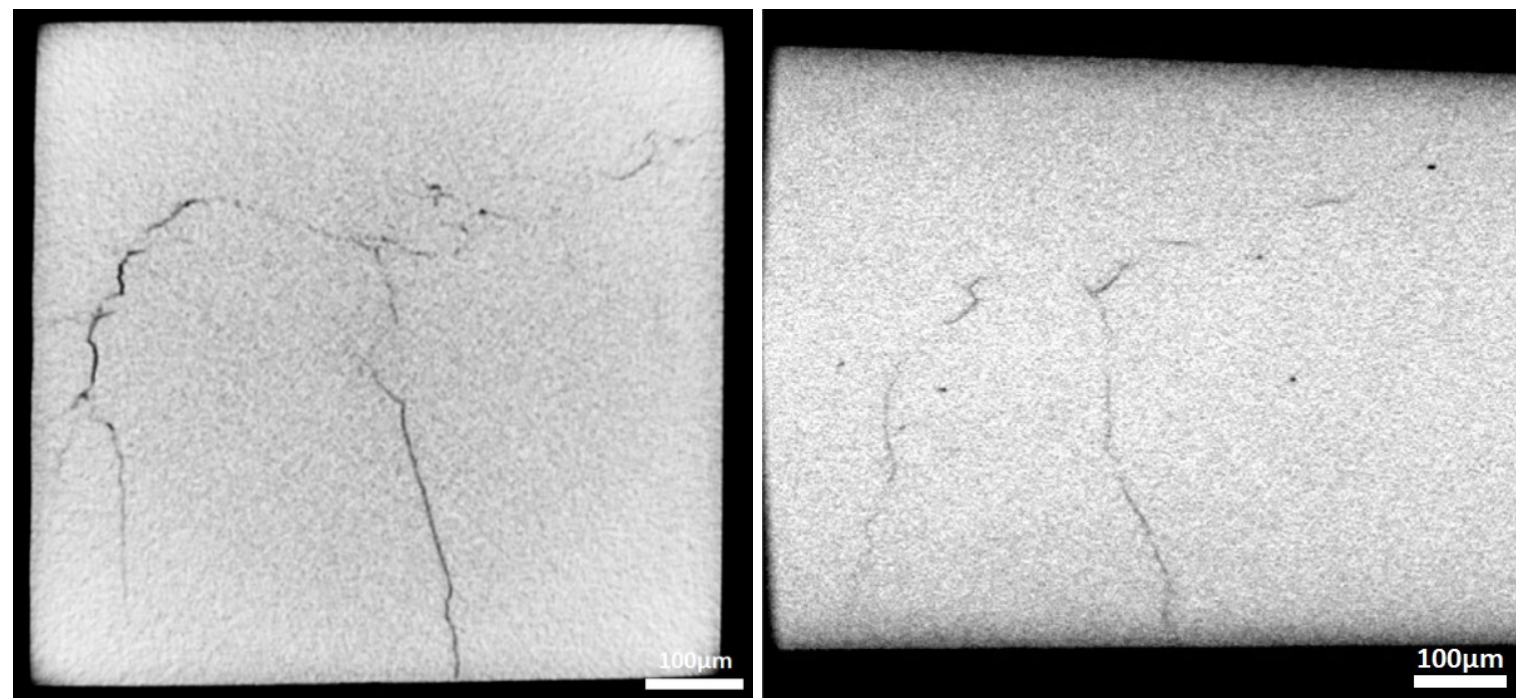

Figure 14: $X$-ray tomography slice of two different irregular WEC networks, viewed along the rolling direction. (a) Taken from specimen A, voxel size is $0.49 \mu \mathrm{m}$, calculated maximum Tresca stress $146 \mu \mathrm{m}$ and critical plane at $85.0^{\circ} \pm 0.7$. (b) Taken from specimen $D$, voxel size is $0.65 \mu \mathrm{m}$, calculated maximum Tresca stress $430 \mu \mathrm{m}$ and critical plane at $85^{\circ} \pm 0.7$.

The XCT technique used in this work yields 3D density maps, which can be used for visualizing the morphology of WEC in the scanned specimen. Simple reconstruction methods have been used for creating 3D maps to demonstrate the potential of the technique, showing it is possible to map out relatively small WEC within the bulk material, such as butterfly-like cracks. Still there are limitations on mapping narrower side branches or crack tips, especially if a global threshold mapping procedure is used for segmentation. In general it was found that the XCT could deliver a resolution close to that of optical microscopy for crack sizes down to $0.5 \mu \mathrm{m}$, the voxel size used in this work was down to $0.49 \mu \mathrm{m}$. While the WEA can be observed in SEM or optical microscopy if etched, it is not possible to distinguish WEA using XCT as the density is the same as for the bulk steel. Inclusions and porosities however are very easy to map using XCT, as they are 3D defects with a significant volume compared to cracks, which normally have a very narrow thickness. Inclusions like $\mathrm{MnS}$ and $\mathrm{Al}_{2} \mathrm{O}_{3}$ can be detected by XCT due to the difference in density from the bulk steel, however if the inclusions are small it is not possible to tell them apart from other 3D defects, such as porosities. This is relevant for WEC, since the crack propagation tends to create cavities next to the crack [26], which cannot be distinguished from smaller inclusions using XCT. However, it was demonstrated in this work that the 3D maps can be used for identifying interesting locations and serve as a guide for sectioning, where EDS chemical analysis then can be used for distinguishing the smaller inclusions from cavities.

There are some limitations for investigating small internal WEC networks. When studying narrow cracks, the investigated specimen cross section needs to be accordingly small. In this work, specimen size with a cross section down to $0.5 \mathrm{~mm} \times 0.5 \mathrm{~mm}$ were used, with the individual scans being less than one cubic millimeter in volume. Several scans from the same specimen can be stitched together, as was done in this work, and if necessary even larger networks could be reconstructed by combining scans of adjacent specimens. The location of WEC needs to be established first using other techniques, as scanning random locations with XCT for WEC would require a lot of effort due to the limited size of the scanned volume In this work optical microscopy was used along with visual inspection, however other techniques such as ultrasonic scans can also be used, as shown in [13]. 
It is not possible to make a general statement regarding the correlation between the depth of the Tresca stress and the location of WEC. The best correlation was found in specimen A, which was tested under constant laboratory conditions. Therefore, it can be assumed that a better correlation would be achieved in specimen $B, C$ and $D$ if the real load and stress history is taken into account. A correlation between the critical plane orientation, which predicts the orientation of the crack initiation, and the morphology of WEC was not found. Therefore, it is assumed that the critical plane orientation does not control the orientation of the WEC propagation.

\section{Conclusion}

In this work it has been demonstrated that 3D mapping of a variety of White Etching Cracks can be achieved using X-ray Computerized Tomography laboratory equipment based on density measurements. This allows detection of small density differences in the bulk material, such as cracks, although it is not possible to map WEA due to similar density to the matrix. WEC networks can be reconstructed as 3D maps using simple global or local threshold procedures, although there are limitations for parts of the cracks narrower than $0.5 \mu \mathrm{m}$, such as crack tips and side-branching cracks. In general, inclusions can be mapped as well but smaller inclusions could not be distinguished from small cavities. It was demonstrated that the 3D maps could be used as a guide for finding features observed in XCT with sectioning techniques, interesting features such as inclusions interacting with the cracks could be investigated using electron microscopy allowing for chemical analysis. It was also demonstrated that the location of WEC correlate with the calculated depth of the maximum Tresca stress when the real load and stress history is known, but not for the wind turbine bearings where the history is not known. It can also by concluded that the propagation direction of WEC is not defined by the calculated critical plane orientation.

\section{Data availability}

The raw/processed data required to reproduce these findings cannot be shared at this time due to technical or time limitations.

\section{References}

[1] Grabulov A, Petrov R, Zandbergen HW: EBSD investigation of the crack initiation and TEM/FIB analyses of the microstructural changes around the cracks formed under Rolling Contact Fatigue (RCF), International Journal of Fatigue 32 (2010) 576-583 DOI:10.1016/j.ijfatigue.2009.07.002

[2] Diederichs AM, Schwedt A, Mayer J, Dreifer T: Electron microscopy analysis of structural changes within White Etching Areas, Materials Science and Technology Volume 32 (2016) 1683-1693

[3] Evans MH: An updated review: white etching cracks (WECs) and axial cracks in wind turbine gearbox bearings, Materials Science and Technology 32 (2016) 1133-1169

DOI:10.1080/02670836.2015.1133022

[4] Gegner J, Nierlich W: Service Loading Analysis of Wind Turbine Gearbox Rolling Bearings Based on X-Ray Diffraction Residual Stress Measurements, Materials Science Forum, 768-769 (2014) 723-732 DOI:10.4028/www.scientific.net/MSF.768-769.723

[5] AR Crehu: Tribological analysis of White Etching Crack (WEC) failures in rolling element bearings, INSA de Lyon, mechanics of materials, PhD Thesis 2014

[6] Evans $\mathrm{MH}$ : White structure flaking failure in bearings under rolling contact fatigue. University of Southampton, Faculty of Engineering and the Environment, PhD Thesis 2013 
[7] Stadler K, Lai J, Vegter RH: A review: The dilemma with premature white etching crack (WEC) bearing failures, BEARING STEELTECHNOLOGIES: 10TH VOLUME, ADVANCES IN STEELTECHNOLOGIES FOR ROLLING BEARINGS, STP 1580, 2014

[8] Hengerer F, Brockmuller U, Sorstom PO: Through-hardening or case-hardening for tapered roller bearings, Creative use of bearings steels, ASTM STP 1195, Hoo JJC ed., American Society for Testing and Materials, Philadelphia 1993, 21-33

[9] Errichello R, Budny R, Eckert R: Investigations of Bearing Failures Associated with White Etching Areas (WEAs) in Wind Turbine Gearboxes, Tribology Transactions 56 (2013) 1069-1076 DOI:10.1080/10402004.2013.823531

[10] Lai J, Stadler K, Investigation on the mechanisms of white etching crack (WEC) formation in rolling contact fatigue and identification of a root cause for bearing premature failure, Wear 364-365 (2016) 244-256

[11] Bruce T, Rounding E, Long H, Dwyer-Joyce RS: Characterisation of white etching crack damage in wind turbine gearbox bearings, Wear 338-339 (2015) 164-177 DOI:10.1016/j.wear.2015.06.008

[12] Evans MH, Richardson AD, Wang L, Wood RJK, Anderson WB: Confirming subsurface initiation at non-metallic inclusions as one mechanism for white etching crack (WEC) formation, Tribology International, 75 (2014) 87-97

[13] Gould B, Greco A, Stadler K, Vegter E, Xiao X: Using advanced tomography techniques to investigate the development of White Etching Cracks in a prematurely failed field bearing, Tribology International 116 (2017) 362-370 DOI:10.1016/j.triboint.2017.07.028

[14] Feldkamp LA, Davis LC, Kress JW, Practical cone-beam algorithm. Journal of the Optical Society of America, A 1(6) (1984), 612-619.

[15] Glasbey CA, an analysis of histogram-based thresholding algorithms, Graphical Models and Image Processing, vol 55, no. 6, pp. 532-537, 1993.

[16] Padilla E., Chawla N., Silva L.F., Santos V.R. dos, Paciornik S., Image analysis of cracks in the weld metal of wet welted steel joint by three dimensional (3d) X-ray microtomography, Materials Characterization 83 (2013) 139-144

[17] Gutiérrez Guzmán F, Oezel M, Jacobs G, Burghardt G, Broeckmann C, Janitzky T: Reproduction of white etching cracks under rolling contact loading on thrust bearing and two-disc test rigs, Wear

390-391 (2017) 23-32

[18] Danielsen HK, Gutiérrez Guzmán F, Dahl KV, Li YJ, Wu J, Jacobs G, Burghardt G, Fæster S, Alimadadi H, Goto S, Raabe D, Petrov R: Multiscale characterization of White Etching Cracks (WEC) in a 100Cr6 bearing from a thrust bearing test rig, Wear 370-371 (2017) 73-82 DOI:10.1016/j.wear.2016.11.016

[19] IEC (2012): IEC 61400 - 4 Wind turbines - Part 4: Design requirements for wind turbine gearboxes 
[20] ISO, DD (2008): TS 16281 Rolling bearings-methods for calculating the modified reference rating life for universally loaded bearings. In International Organization for Standardization, Geneva, Switzerland.

[21] Gegner J: Tribological Aspects of Rolling Bearing Failures, DOI: 10.5772/20790

[22] Polonsky IA, Keer LM: A numerical method for solving rough contact problems based on the multi-level multi-summation and conjugate gradient techniques, Wear 231 (1999) 206-219

DOI:10.1016/S0043-1648(99)00113-1

[23] Liu S, Wang Q: Studying Contact Stress Fields Caused by Surface Tractions With a Discrete Convolution and Fast Fourier Transform Algorithm, J. Tribol. 124 (2002) 36-45 DOI:10.1115/1.1401017.

[24] Fatemi A, Socie DF: A critical plane approach to multiaxial fatigue damage including out-ofphase loading, Fat Frac Eng Mat Struct 11 (1988) 149-165 DOI:10.1111/j.1460-2695.1988.tb01169.x.

[25] Bernasconi A, Papadopoulos IV: Efficiency of algorithms for shear stress amplitude calculation in critical plane class fatigue criteria, Computational Materials Science 34 (2005) 355-368 DOI:10.1016/j.commatsci.2005.01.005.

[26] Harada H, Mikami T, Shibata M, Sokai D, Yamamoto A, Tsubakino H: Microstructural changes and crack initiation with white etching area formation under rolling/sliding contact in bearing steel, ISIJ International, 45 (2005) 1897-1902 